\title{
Auswahl und Aufbau eines Multisensor-Fernmesssystems zur Fluidleck- erkennung für den Einsatz auf einem mobilen Industrie-Inspektionsroboter
}

\author{
Samuel Soldan, Gero Bonow, Andreas Kroll \\ Universität Kassel, Fachbereich Maschinenbau \\ Fachgebiet Mess- und Regelungstechnik \\ Mönchebergstraße 7, 34125 Kassel \\ Telefon: 05618041826 \\ E-Mail: \{samuel.soldan, gero.bonow, andreas.kroll\}@mrt.uni-kassel.de
}

\section{Kurzfassung}

Eine regelmäßige und sorgfältige Inspektion von druckführenden Komponenten in Industrieanlagen (z. B. Mineralölraffinerien oder Chemieanlagen) ermöglicht ein frühzeitiges Erkennen von möglichen Störungen und Defekten und gewährleistet dadurch eine Betriebssicherheit auf hohem Niveau. Routineinspektionen werden oft nur mit einfachen lokal messenden Gasmessgeräten durchgeführt und sind durch wechselnde Inspekteure und Schichtarbeit personen- und tagesformabhängig. Dabei ist die Inspektionstätigkeit zumeist monoton und ermüdend. Die hohen Anschaffungskosten, die notwendige umfangreiche Einarbeitung in die Bedienung, die niedrige Auslastung bei manueller Verwendung sowie das schwer quantifizierbare Einsparungspotential schrecken oft von der Beschaffung von Fernmessgeräten ab.

Ziel des Projektes RoboGas ${ }^{\text {Inspector }}$ [1] ist die Entwicklung eines mobilen, mit infrarotoptischer Fernmesstechnik ausgestatteten professionellen Service-Roboters für die Routineinspektion von Industrieanlagen. Durch den Einsatz auf einem Roboter kann die Messtechnik voll ausgelastet werden, da dieser rund um die Uhr im Betrieb sein kann. Der Mensch wird von eintöniger Arbeit entlastet und das Fachpersonal steht für anspruchsvollere Aufgaben zur Verfügung. Die Reproduzierbarkeit der Ergebnisse wird verbessert und lässt zudem eine präzisere Differentialdiagnose mit historischen Daten zu; dadurch kann auch der Schädigungsverlauf dokumentiert werden. Durch die Verwendung von Messgeräten mit verschiedenen Messprinzipien kann eine Leckhypothese abgesichert und die Wahrscheinlichkeit von Falschalarmen reduziert werden.

Die Interoperabilität der verschiedenen Messgeräte und deren modularer, mechanischer und elektrischer, spritzwassergeschützter Aufbau auf einer mobilen Roboterplattform wird in diesem Beitrag diskutiert und mit praktischen Erfahrungen zur Leistungsfähigkeit des Systems abgerundet. Dazu wurde ein modulares Messsystem entworfen, prototypisch realisiert und im Labor getestet. Das System verfügt über eigene Rechenkapazität und fasst die unterschiedlichen Schnittstellen sowie die Messdatenerfassung der Messgeräte zusammen. Dies vereinfacht somit die Verwendung des Systems auf verschiedenen Roboterplattformen.

\section{Motivation}

Für Betreiber prozesstechnischer Anlagen und Versorgungsinfrastruktur ist es eine kontinuierliche Aufgabe, die Betriebssicherheit auf hohem Niveau zu gewährleisten und den gesetzlichen Vorgaben zu genügen. Hierzu werden meist Präventions- und Inspektionsprogramme etabliert, die zeitbeanspruchende, vom Menschen täglich durchgeführte Routineinspektionsgänge einschließen. Aus mehreren Gründenist die Erkennung von Lecks ein wichtiger Aufgabenbereich bei diesen Routineinspektionen: so können die austretenden Stoffe eine Gefahr für den Menschen und die Umwelt darstellen, einen finanziellen Verlust für den Anlagenbetreiber bedeuten aber auch einen größeren Folgeschaden ankündigen (Stichwort Leckvor-Bruch[2]).

Dabei arbeitet der Inspekteur oft ohne, oder mit einfacher lokal messenderGasmesstechnik und nutzt seine Sinneswahrnehmung sowie persönliche Erfahrungen. Eine Ausnahme ist in bestimmten Inspektionsszenarien die Infrarot-Thermografie,welche zu einer verbreiteten Technik geworden ist. Hierbei sind die Anwendungsbereiche besonders die Inspektion von elektrischen (Überlast, Kurzschlüsse), mechanischen (Reibung, Abnutzung, Verstopfungen von Ventilen oder Rohren)oder baulichen Einrichtungen (Isolierung, Feuchtigkeit, Lecks)[3], [4].

Neuartige Fermesstechniken erlauben eine Detektion von Gasen aus Entfernungen bis zu mehreren Kilometern. Damit lassen sich große Bereiche zügig überprüfen und Komponenten in abgelegenen oder schwer zu erreichenden Orten leicht inspizieren. Auch für Flächenquellen sind Fernmesstechniken sehr interessant. Verschiedene Messmethoden und -geräte sind am Markt verfügbar[5]. Durch ihren verhältnismäßig hohen Anschaffungspreis und die Komplexität der Bedienung werden diese Geräte jedoch nur sehr selten eingesetzt[6]. 
Die Entwicklung neuartiger Überwachungsverfahren, welche die Möglichkeiten modernster Mess-, Automatisierungs- und Robotertechnik ausschöpfen, versprechen eine erhöhte Güte und Wirtschaftlichkeit der Inspektionen bei gleichzeitiger Entlastung des Menschen von monotonen, zeitaufwändigen Tätigkeiten. Das Projekt RoboGas ${ }^{\text {Inspector }}$ verfolgt deshalb das Ziel, ein innovatives Mensch-Maschine-System mit intelligenten, kooperierenden und mit Gasfernmesstechnik ausgestatteten Inspektionsrobotern zu entwickeln, das frühzeitig Lecks entdecken kann. In diesem System kann die Überwachung von Anlagen sowie die Detektion und Ortung von Lecks weitgehend autonom von mobilen Robotern bewältigt werden, die zugleich die Auswertung der gemessenen Daten und die Dokumentation der Inspektionen übernehmen. Dadurch sollen Verbesserungen in drei Bereichen adressiert werden:

- Inspektionsgüte: Gleichbleibend hohe Inspektionsqualität durch Automatisierung; Prüfvorgang verbessern mittels mobiler Gasfernmesstechnik; lokal verfügbares Wissen durch zentrale Wissensbasis erhöhen; bessere Abdeckung weitläufiger Inspektionsgebiete und Verkürzung der Inspektionsintervalle mit vorhandenem Personal.

- Entlastung des Menschen: Einfachere Inspektion schwer erreichbarer Orte mittels Fernmesstechnik; Automatisierung hochgradig repetitiver, monotoner Routineaufgaben; Automatisierung von Auswertung und Dokumentation.

- Wirtschaftlichkeit: Effizienter und effektiver Einsatz verfügbarer Fachkräfte; Bedien- und Überwachungspersonal Freiräume für Tätigkeiten mit höherer Wertschöpfung verschaffen wie Planung, leitende Kontrolle, Optimierung; effizienter Einsatz hochwertiger Messgeräte.

Die dabei eingesetzte Messtechnik besteht aus mehreren Sensoren

a) zur Detektion von unterschiedlichen Gasen,

b) Validierung von Leckhypothesen mit verschiedenen Sensoren (Robustheit),

c) Entwicklung und Untersuchung von unterschiedlichen Leckdetektionsverfahren (Forschung) und

d) Ortung der Leckquelle.

In diesem Beitrag werden zunächst die Messaufgabe, die Grundlagen und die Auswahl von Messgeräten für ein mobiles, multisensorisches Fernmesssystem zur Detektion von Gas- und Flüssigkeitsleckagen in industriellen Anlagen und Einrichtungen beschrieben. Dabei werden unterschiedliche Messverfahren vorgestellt und deren Anwendung und Kombinationsmöglichkeiten bei der Ortung von Fluidleckagen erläutert. Die Integration der Messgeräte in ein einfach umrüst- und integrierbares Inspektionsmodul wird anschließend diskutiert und die Leistungsfähigkeit des Systems vorgestellt.

\section{Messaufgabe}

Die automatische Inspektion von Anlagenbereichen mit Hilfe von mobilen Robotern ist insbesondere in

- chemischen und petrochemischen Komplexen sowie

- Infrastruktur für die Erdgasdistribution (Verteiler/Verdichterstationen, Pipelines) und

- geschlossene Abfalldeponien

aufgrund der Anlagengrößen interessant (siehe Abbildung 1). Bei den petrochemischen Anlagen sind Gase aus der homologen Reihe der Alkane (Methan, Ethan, Propan, Butan, Pentan, Hexan, Heptan, Oktan) aber auch andere Gase wie Schwefelhexafluorid oder Amoniak für die Detektion von Interesse.Da Erdgas zu einem großen Teil aus Methan besteht, ist dessen messtechnische Erfassung für den Bereich der Erdgasdistributionsinfrastruktur gefordert. In Abfalldeponien entstehen durch den chemischen und bakteriologischen Abbau von organischen Inhaltsstoffen des Abfalls hauptsächlich Methan und Kohlenstoffdioxid. Die Deponiegase werden normalerweise gesammelt und abgefackelt oder für die Erzeugung von Strom oder Wärme verwendet. Halbjährig Überprüfungen über die Wirksamkeit der Deponiegaserfassung werden in diesem Bereich sogar durch den Gesetzgeber vorgeschrieben[7].

Mehrere Anlagenbegehungen und Interviews mit Inspekteuren wurden durchgeführt in denen typische Inspektionsziele (Lage, Form, Art, usw.) ermittelt, besichtigt und vermessen wurden. Im Kontext der Inspektion von Anlagen sind typische Leckorte überwiegend Ventile, Pumpen, Stopfbuchsen, Flansche sowie Rohrauflager und Dehnungsentlastungstellen. Rohrtrassen und -brücken bzw. Verteilstellen, Prozessanlagen und Tanklager (z. B. diffuse Emissionen aus Schwimmdachtanks [8]) mit solchen Komponenten sind demnach die primären Inspektionsziele (Abbildung 2). In den Abfalldeponien sollen sowohl Gasbrunnen als auch die Versiegelungsschicht (meist mit Rasen bepflanzt) flächig inspiziert werden. Die zu inspizierenden Komponenten liegen bis zu $10 \mathrm{~m}$ von einem befahrbaren Weg/Untergrund entfernt und können sich über eine Tiefe in Anlagen von $15 \mathrm{~m}$ erstrecken (z. B. Abbildung 2, unten links).

Die häufigste Leckursache ist, neben alternden Dichtungen, auch Kontaktkorrosion, die sowohl an der Rohraußenseite (z. B. Kontaktkorrosion an Rohrauflagern) als auch an der Rohrinnenseite (z. B. durch Produktablagerungen oder Abrasion) auftreten kann. Aufgrund der möglicherweise schlecht einsehbaren Lage oder durch äußere Verschmutzungen können solche Beschädigungen aber bei herkömmlicher Inspektion zum Teil erst bei einem deutlich sichtbarenProduktaustritt erkannt werden. 

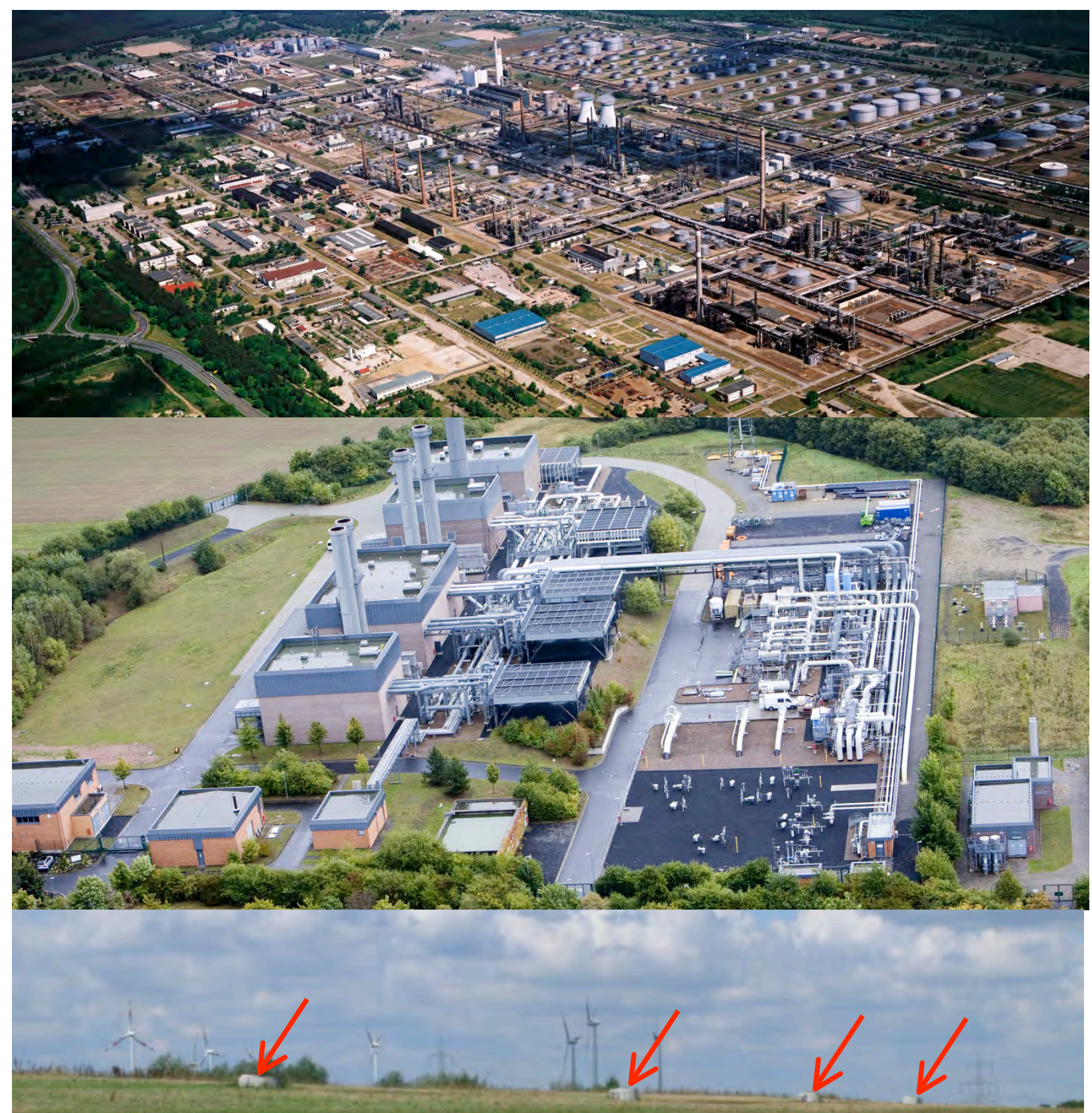

Abbildung 1: Oben: Luftbild einer Raffinerie[@ PCK Raffinerie $\mathrm{GmbH}$ ], Mitte: Luftbild einer Gasverdichterstation [@ WINGAS TRANSPORT GmbH], Unten: geschlossene Abfalldeponie mit Gasbrunnen (Pfeile).

Die Inspektion erfolgt im Freien und soll zu jeder Tages- und Jahreszeit möglich sein. Bei besonders schlechtem Wetter (starker Regen/Schnee, dichter Nebel) muss eine Inspektion allerdings nicht gewährleistet sein, da ausphysikalischen Gründen eine optische Gasfernmessung dann nur bedingt möglich ist.Trotz alledemsollten die verwendeten Messgeräte und Bauteile widrigeren Wetterbedingungen schadlos ausgesetzt werden können. Kritische Gebäudeinnenbereiche werden hier nicht betrachtet, da sie typischerweise mit Gasmeldeanlagen ausgestattet sind. Diese ermöglichen zwar keine Leckortung aber 
eine Detektion, sobald eine Leckage zu einer ausreichend hohen Gaskonzentration am Gefahrenmelder führt.

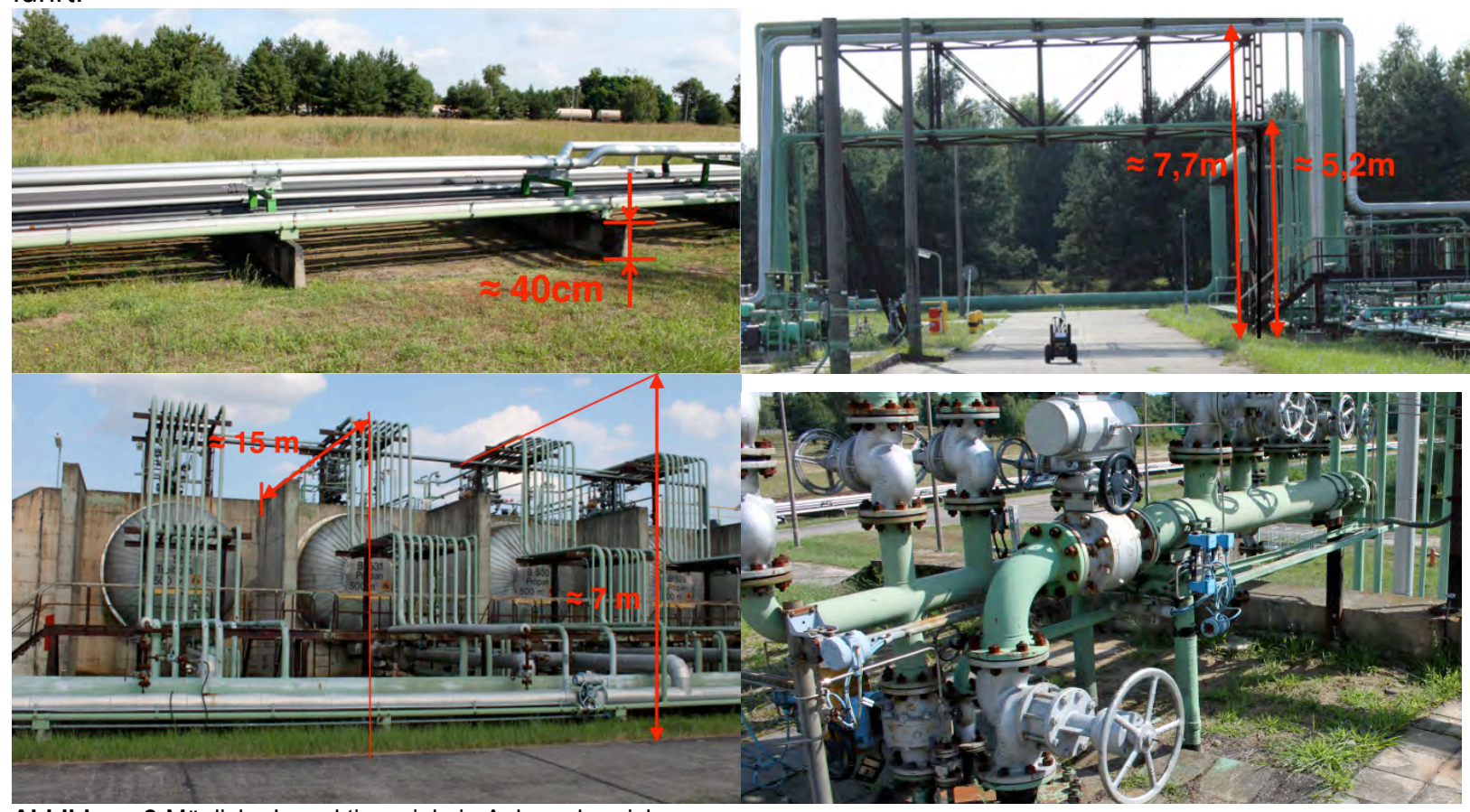

Abbildung 2:Mögliche Inspektionsziele in Anlagenbereichen.

Eine geeignete Vorrichtung zum Schwenken und Neigen der Messgeräte ist notwendig, da die Inspektionsziele sowohl flächig, als auch oberhalb des Roboters sein können. Die Schwenkgeschwindigkeiten sollten sich dabei an den Messfrequenzen der Messgeräte orientieren, falls während der Bewegung gemessen werden soll und nicht nur im Stand.

Um die Entwicklung und Erprobung von Messstrategien zu vereinfachen,soll sich das MultisensorFernmesssystem einfach auf verschiedenen Roboterplattformen einsetzen lassen (z. B. Roboter für Laboreinsatz und Roboter für Freifeldumgebungen). Ein modularer Einsatz von Messgeräten ist erwünscht,um das System einfach auf die Zielsubstanzen in verschiedenen zu inspizierenden Anlagen einstellen zu können. Während der Systementwicklung lassen sich so zudem einfach verschiedene Sensorsysteme austauschen/kombinieren. Für Voruntersuchungen ist zudem eine Stand-AloneBetreibbarkeit des Messsystems ohne Roboterplattform notwendig, um z. B. unter Laborbedingungen Performance-Untersuchungen genau und reproduzierbar durchführen zu können. Das Messsystem soll daher über eigene Rechenkapazitäten verfügen und nicht direkt von der Sensorik der Roboterplattform (z. B. Frontlaserscanner) abhängig sein.

\section{Grundlagen}

ZurDetektion von Gasen und Ortung vonLecks aus der Entfernung können verschiedene Messeffekte ausgenutzt werden. Zum einen können die Absorptionseigenschaften der Gase bei elektromagnetischer Strahlung ausgenutzt werden, die im direkten Zusammenhang mit Spezies und Konzentration der Gase oder Gasgemische stehen (Primäreffekt). Zum anderen können auch Sekundäreffekte, wie Schallemissionen (z. B. Ultraschall), Abkühleffekte bzw. Vereisungen am Lecksowie Vegetationsschäden in Lecknähegenutzt werden. Im Folgenden sollen jedoch nur optische Messverfahren betrachtet werden.

Grundsätzlich tauscht jeder Körper mit seiner Umgebung (abhängig von seiner Temperatur) Energie durch Strahlung mit unterschiedlichen Spektren aus. Wie stark ein Körper mit elektromagnetischer Strahlung interagieren kann, ist dabei stark vom Material abhängig. Während ein idealer Spiegel sämtliche Strahlung reflektiert, ein ideales Fenster Strahlung vollständigtransmittiert und die eintreffende Strahlung von einem Schwarzen Körper vollständig absorbiert wird, besitzen natürliche Materialien diese Eigenschaften in unterschiedlich starker Ausprägung, die zudem auch von der Wellenlänge und der Temperatur abhängig sind. Auch Gase besitzen diese Eigenschaften, jedoch mit stärker ausgeprägten Transmissions- und Absorptionseigenschaften.

Durchläuft eine Strahlung mit einem bekannten Emissionsspektrum $I_{0}(\lambda)$ ein Gas und wird anschließend das Spektrum $I_{M}(\lambda)$ der am Detektor registrierten Strahlung analysiert, so fällt durch den Vergleich der Spektren auf, dass bei bestimmten Wellenlängen Abschwächungen in den Strahlungsintensitäten auftre- 
ten (vgl. Abbildung 3). Hierbei kann sowohl eine natürliche (z. B. Sonne, Hintergrundstrahlung), als auch künstliche Strahlungsquelle (z. B. Laser) verwendet werden.

Wie viel Strahlungsenergie im Endeffekt wellenlängenabhängig absorbiert wird, ist vom atomaren und molekularen Aufbau des Gases abhängig und somit für jedes Gas unterschiedlich.Für die optische Fernmesstechnik stehen nicht beliebige Wellenlängenbereiche zur Verfügung, da auch die Atmosphäre in vielen Bereichen starke Absorptionen aufweist (hauptsächlich wegen Wasser und $\mathrm{CO}_{2}$ ). Aus diesem Grund werden für die optische Fernmesstechnik bevorzugt Wellenlängenbereiche gewählt, in denen die Atmosphärengase keine oder vernachlässigbare Absorptionen aufweisen. Diese Bereiche werden auch als „Atmosphärische Fenster" bezeichnet und liegen etwa zwischen 1,25 $\mu \mathrm{m}$ und $5 \mu \mathrm{m}$ sowie $7 \mu \mathrm{m}$ und $14 \mu \mathrm{m}[4]$. Aufgrund der Absorptionscharakteristiken vieler technischer Gase im ersten Spektralbereich wird dieser bevorzugt für optische Gasfernmesssysteme genutzt. Für die Thermografie ist der längerwellige Bereich vorteilhafter, da ein größerer spektraler Bereich für die Messungen verwendet werden kann, in dem zudem für Umgebungstemperaturen auch die Maxima der Strahlungsintensitäten liegen (siehe Abbildung 4).

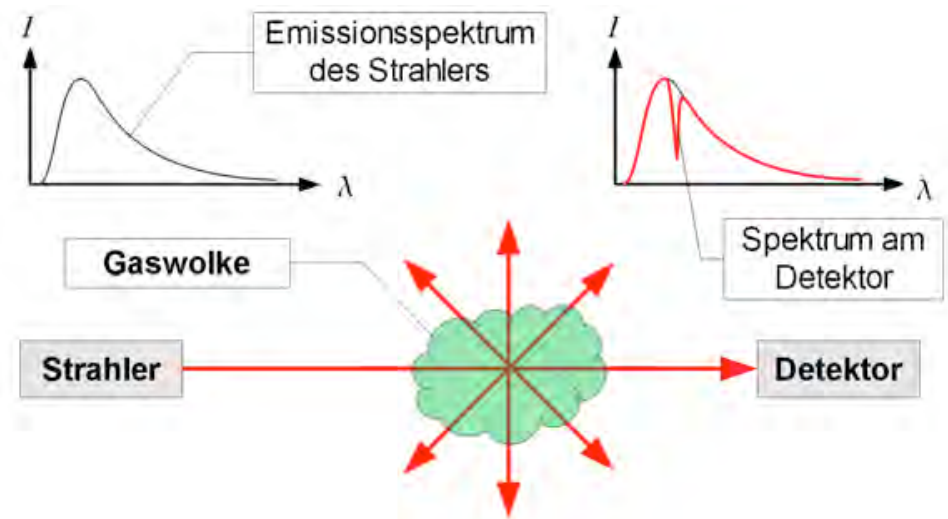

Abbildung 3:Beispiel für die Gasfernmessung auf der Grundlage der Absorption elektromagnetischer Strahlung: Vom eingestrahlten Emissionsspektrum der Strahlungsquelle wird vom Gas konzentrationsabhängig ein Teil der Strahlung absorbiert. Durch den Vergleich von emittierten und detektierten Spektren kann a) die Präsenz des Gases nachgewiesen und b) die Konzentration im Messpfadkumulativ bestimmt werden.

Zur Temperaturfernmessung wird der Betrag der von einer Oberfläche abgestrahlten bzw. emittierten Strahlungsleistung gemessen. Als Referenz wird die spektrale Verteilung von einem idealen Schwarzen Körper verwendet, die über das Planksche Strahlungsgesetzt bestimmt und die Maximalwerte in Abhängigkeit von der Objekttemperatur über die Wiensche Verschiebungsfunktion berechnet werden kann (vgl. Abbildung 4).

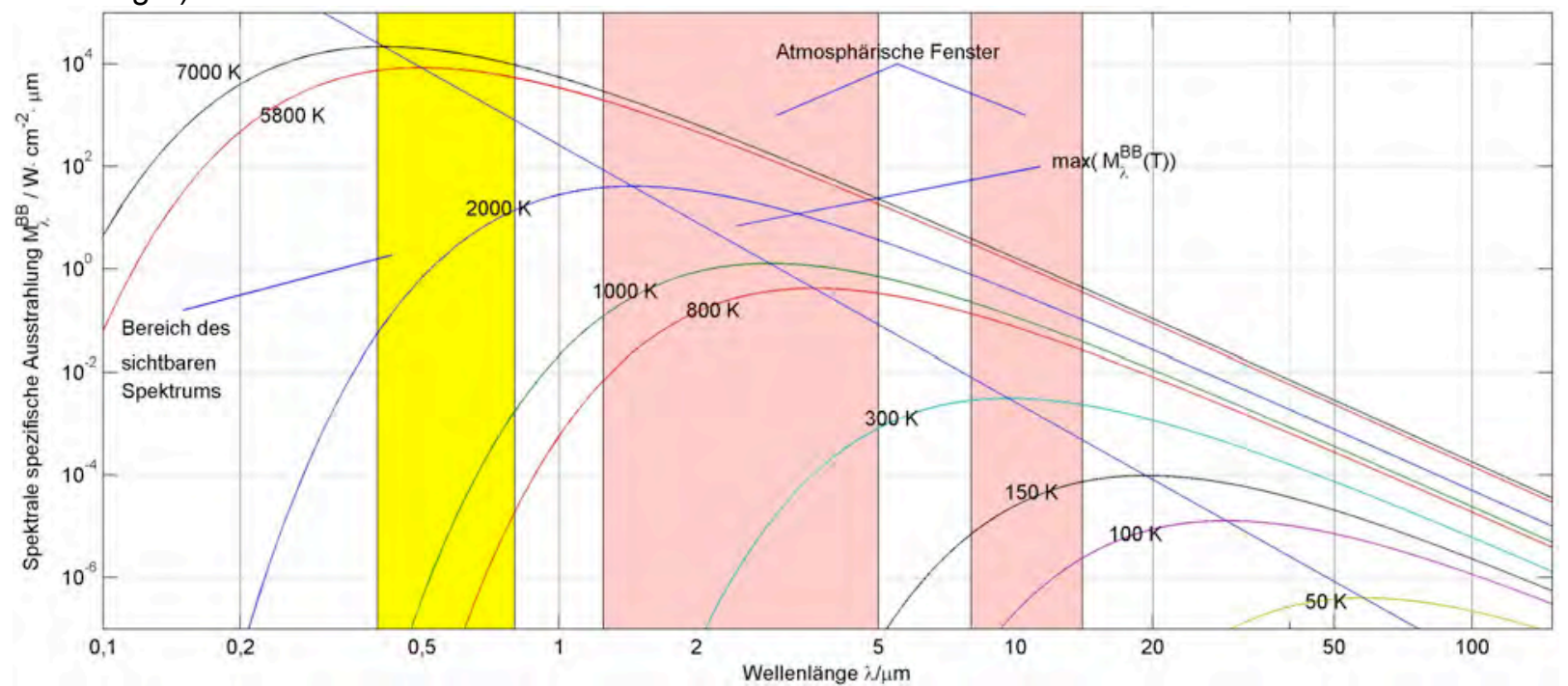

Abbildung 4: Spektrum der spezifischen Ausstrahlung eines idealen schwarzen Körpers für verschiedene Temperaturen nach dem Planckschen Strahlungsgesetz(nach [4]).

Durch den Vergleich der gemessenen Strahlungsleistung mit der Strahlungsleistung eines idealen Strahlers (Schwarzer Körper) kann die Temperatur des betrachteten Objekts errechnet werdenwerden, sofern die Sensoreigenschaften und Eigenschaften der Messstecke sowie des betrachteten Objekts berücksichtigt werden. Problematisch sind hierbei die signifikant unterschiedlichen Emissions-, Reflexions- und 
Transmissionseigenschaften verschiedener Materialien. Insbesondere Metalle weisen im infraroten Spektralbereich einen sehr hohen Reflexionsgrad auf, wodurch eine Temperaturmessung massiv verfälscht werden kann, wenn der Emissionsgrad falsch gewählt wird.

Auf der anderen Seite kann wenn das wellenlängenabhängige Absorptionsverhalten $\alpha(\lambda)$ eines Gases bekannt ist durch eine Spektralanalyse der Strahlungsintensitäten und mittels Mustererkennungsverfahren das Gas im Messpfad identifiziert werden. Neben der Identifikation des Gases besteht auch die Möglichkeit die mittlere Gaskonzentration $\bar{C}_{\text {Gas }}$ im Messpfad zu bestimmen, da die Absorption zum einen von der Messpfadlänge im Gas ${ }^{L}$ Pfad und zum anderen von der lokalen Gaskonzentration im Messpfad abhängig ist. Dieser Zusammenhang wird durch das Lambert-Beer-Gesetz beschrieben [9]:

$I_{M}(\lambda)=I_{0}(\lambda) \cdot \exp \left(-\alpha(\lambda) \cdot \int_{x=0}^{L} C_{s}(x) \mathrm{d} \mathbf{x}\right)$

Häufig ist die Messpfadlänge nicht bekannt. Aus diesem Grund wird beim Einsatz von Gasfernmessgerä-

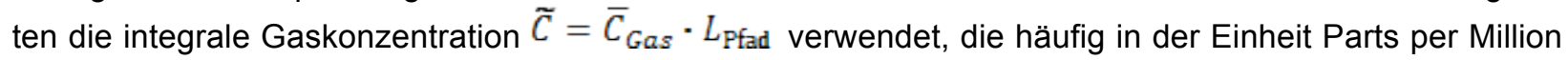
Meter $(\mathrm{ppm} \cdot \mathrm{m})$ bzw. Parts per Billon Meter $(\mathrm{ppb} \cdot \mathrm{m})$ angegeben wird.

\section{Ausgewählte Messgeräte: Übersicht, Leistungsdaten und Messstrategien}

Nach einer Marktrecherchewurden drei Messgeräte zur Gasfernmessung bzw. -detektionausgewählt[5]:

1. ein aktives spektroskopisches Methanmessgerät auf Basis der Tunable Diode Laser Absorption Spectroscopy (TDLAS),

2. eine Thermografiekamera zur Visualisierung von Gasen (Imaging Spectrometer - IS) und

3. eine Thermografiekamera zur Detektion von Temperaturfeldstörungen durch ein Leck (z. B. Expansionskälte, Verdunstungskälte, von Umgebung abweichende Temperatur ausgetretener Flüssigkeiten).

Weiterhin wurden zur Unterstützung der Messstrategien noch

- ein 2D TOF Laserentfernungsmesser (Laserscanner) und

- eine Videokamera ausgewählt.

Bei TDLAS basierten Gasfernmessgeräten wird ein IR Laser eingesetzt, der in einem kleinen Bereich in seiner Wellenlänge verstimmt werden kann $(\approx \pm 10 \%$ der Nennwellenlänge). Der Laser wird dabei so ausgewählt, dass eine Wellenlänge eingestellt werden kann, bei der das Gas keine oder vernachlässigbare Absorptionen aufweist. Zur Bestimmung der Gaskonzentration nach dem Lambert-Beer-Gesetz muss zudem die zweite Wellenlänge in einem Spektralbereich liegen, bei der das Zielgas eine signifikante Absorption erzeugt. Durch diesen Ansatz wird bei der ersten Messung der Einfluss der Messstrecke und bei der zweite Messung zudem auch der Einfluss des Gases erfasst. Ist der Absorptionskoeffizient bei den Wellenlängen bekannt, kann schlussendlich die integrale Gaskonzentration bestimmt werden. Ist zudem die Messpfadlänge bekannt, kann auch die mittlere Gaskonzentration berechnet werden. TDLAS basierende Gasfernmessgeräte können hierbei in monostatischer (Sender und Empfänger befinden sich in einem Gehäuse) oder bistatischer Messanordnung (Sender und Empfänger stehen sich räumlich getrennt gegenüber) verwendet werden. Die Ansprechschwellen bei TDLAS basierten Gasfernmessgeräten liegt, abhängig vom Gas, in der Größenordnung von $\mathrm{ppm} \cdot \mathrm{m}$ bis $\mathrm{ppb} \cdot \mathrm{m}[6]$. Das ausgewählte TDLAS Gerät zur Ferndetektion von Methan (Sewerin RMLD - [10]) arbeitet im Wellenlängenbereich um 1,6 $\mu \mathrm{m}$ in einer monostatischen Anordnung mit einer Messfrequenz von $10 \mathrm{~Hz}$. Es werden keine speziellen Reflektoren benötigt. Je nach Hintergrund können dabei Messpfadlängen von bis zu $30 \mathrm{~m}$ sowie eine Empfindlichkeit von $5 \mathrm{ppm} \cdot \mathrm{m}$ erreicht werden[11], [12].

Durch einen schmalbandigen Bandpassfilter kann eine Thermografiekamera zur Visualisierung von Gasen verwendet werden[13]. Der spektrale Messbereich der Thermografiekamera wird dabei weitgehend auf die Absorptionsbanden der Zielgase mittels spezieller optischer Filter begrenzt. Für die Kamera ist das Gas somit nicht transparent und sie misst nicht durch das Gas hindurch, sondern ermittelt die Temperatur des Gases. Durch Betrachtung der gewonnen Bilderserie kann dadurch die Gaswolke leicht als Schleier vor dem Hintergrund identifizieren werden, sofern eine ausreichende Temperaturdifferenz zwischen Gas und Hintergrund besteht; eine quantitative Messung sowie eine Unterscheidung von verschiedenen Gasen ist allerdings nicht möglich. Die eingesetzte Kamera (FLIR GF320 - [16]) arbeitet im Spektralbereich von 3,2 $\mu \mathrm{m}$ bis 3,4 $\mu \mathrm{m}$ und hat eine Auflösung von $320 \times 240$ Pixeln. Die Messfrequenz beträgt $60 \mathrm{~Hz}$ (für Video Darstellung im Display oder über HDMI Ausgang), allerdings begrenzt die Datenrate der USB 2.0 Schnittstelle bei Temperaturrohdaten die Messfrequenz auf ca. $4 \mathrm{~Hz}$. Mit diesem Messgerät lassen sich u. a. Benzol, Ethanol, Ethylbenzol, Heptan, Hexan, Isopren, Methanol, MEK, MIBK, Oktan, Pentan, 1-Penten, Toluen, Xylen, Butan, Ethan, Methan, Propan, Ethylen undPropylen detektieren[14], [15]. 
Um minimale Änderungen im Temperaturprofil messen zu können wurdeeine Thermografiekamera (InfratecVarioCamhrhead-[17]) mit einer thermischen Auflösung von besser als 0,03 K und einem spektralen Messbereich von $7,5 \mu \mathrm{m}$ bis $14 \mu \mathrm{m}$ ausgewählt. Mittels GigabitEthernetoder FirewireSchnittstelle können hier Temperaturmatrizen mit 640 x 480 Pixeln bei $60 \mathrm{~Hz}$ ausgelesen werden.

Durch die verschiedenen Messprinzipien unterscheiden sich auch die Messstrategien erheblich. Da ein TDLAS Messgerät nur in eine Richtung misst, muss das Messgerät geschwenkt und geneigt werden, um einen größeren Bereich erfassen zu können. Da der verwendete Laserscanner (SICK LMS151) eine deutlich höhere Messfrequenz hat, können zuvor gewonnen Entfernungsdaten genutzt werden, um Bereiche auszuschließen die zu weit entfernt sind. Weiterhin können die Entfernungsdaten dazu verwendet werden, um die mittlere Gaskonzentration auf der Basis der integralen Gaskonzentration zu berechnen [18]. Sollte nun die Richtung der maximalen Konzentration feststehen, ist eine Messung von mindestens einer anderen Position aus notwendig, um die Position der maximalen Konzentration eindeutig festzustellen (die Gaswolke kann sich ja irgendwo zwischen Messgerät und reflektierendem Objekt befinden). Für die Positionierung für Folgemessungen und die Ortung der Gasquelle können z. B. Triangulationstechnikenverfolgt werden oder sukzessive die Richtung der maximalen integralen/mittleren Gaskonzentration [19].

Bei den bildgebenden Verfahren besteht zunächst keine Veranlassung die Messgeräte zu bewegen. Dies ist lediglich dann notwendig, wenn der zu inspizierende Bereich größer ist als das Sichtfeld der Kameraoptiken oder die Kameras in die falsche Richtung ausgerichtet sind. Bei der Thermografiekamera zur Visualisierung von Gasen ist eine Gasdetektion in Einzelbildern nicht möglich wenn keine Referenzmessung zur Verfügung steht. Die Referenzmessung kann entweder durch weitere Messungen mit dieser oder mit einer anderen Thermografiekamera im ähnlichen Wellenlängenbereich geschehen. Durch den Vergleich der Messungen können nun Bereiche identifiziert werden, in denen zwischen den Einzelmessungen eine hohe Varianz der Messwerte für einen Punkt auftreten. Alternativ ist auch die Erkennung von Bewegungen der Gaswolke möglich, die durch Luftströmungen verursacht werden. Durch die Überprüfung ob im normalen Videobild Bewegungen vorliegen (z. B. ob sich Objekte durchs Bild bewegen), können Falschalarme reduziert werden.

Die Wärmebilder der Thermografiekamera können zunächst durch Vorverarbeitungsschritte wie z. B. Glättung oder Erweiterung der Schärfentiefe verbessert werden. Anschließend kann eine Bildregistrierung von Tiefenkarten und Videobildern helfen, relevante Bildbereiche zu separieren. In diesen Bereichen können dann durch Mustererkennungsverfahren, z. B. durch Korrelation mit idealisierten Leckmustern,Temperaturfeldstörungen durch Lecks oder Undichtigkeiten automatisch gefunden werden[20], [21].

\section{Aufbau und Leistungsfähigkeit}

Basierend auf den Messaufgaben und den ausgewählten Messgeräten wurde ein Inspektionsmodul entworfen und realisiert (Abbildung 5). Das zentrale Aktorelement ist dabei eine Schwenk-Neige-Einheit (PTU - Pan-Tilt Unit) der Firma Schunk (Modell PW90[22]). Damit lassen sich die Messgeräte ausrichten, ohne dass der Roboter bewegt werden muss. Mit einer Winkelgeschwindigkeit von150\% (Achse 1)bzw. $240 \%$ s (Achse 2) und bei Beschleunigungen von600\% $/ \mathrm{s}^{2}$ (Achse 1) und $960 \% / \mathrm{s}^{2}$ (Achse 2) können auch größere Bereiche problemlos und zügig inspiziert werden. Dabei liegt die Wiederholgenauigkeit von Positionieraufgaben bei jeder Achse bei $0,04^{\circ}$.

Die Anbauorientierung der Schwenk-Neige-Einheit ist nicht vorgeschrieben; die PTU wurde auf dem Kopf eingebaut weil das Drehmoment der Achsen unterschiedlich und bei Achse 1 mit $23 \mathrm{Nm}$ höher ist als bei Achse 2 mit $12 \mathrm{Nm}$. Für die gewählte Einbauart wird so nur ein sehr geringes Drehmoment für eine Schwenkbewegung benötigt und für die Neigung ein größeres - entsprechend der verfügbaren Drehmomente des Aktors. Bei einem Stromausfall verhindert zudem eine mechanische Bremse an Achse 1 eine ungewollte Bewegung und mögliche Beschädigungen der Messgeräte oder Anschlussleitungen. Weiterhin vermessen die Messgeräte bei dieser Anordnungund beim Schwenken (Rotation von Achse 2) den Bereich in einem äquidistanten Kreis um das Messmodul auch wenn die Messgeräte durch die andere Achse (Achse 1)zuvor geneigt wurden. Bei der umgekehrten Anbauvariante wäre dazu eine komplexere Kompensationsbewegung von beiden Achsen notwendig und die Kinematik wäre weniger transparent.

Die Verteilung der Messgeräte auf der PTU wurde stark durch den Entfernungsmesser geprägt: durch den $270^{\circ}$-Messbereich war es nicht möglich, diesen an der Seite der PTU zu montieren. Auch die Anschlusskabel sind sehr ausladend so dass der Laserscanner oberhalb der PTU angebracht wurde. Bei den übrigen Komponenten wurde versucht die Lasten möglichst nah an der Neigeachse (Achse 1) zu montieren um das Massenträgheitsmoment und den Energieverbrauch gering zu halten. Da die PTU nicht symmetrisch ist, wurde die Thermografiekamera und das RMLD so links und rechts von der PTU montiert, dass der Schwenkbereich und die im Montagepunkt zur PTU angreifenden Momente minimiert wurden. In einer weiteren Ausbaustufe wurde oberhalb des Laserentfernungsmessers noch eine weitere Montageebene eingebaut um die Gaskamera zu befestigen und Erweiterungsmöglichkeiten zu schaffen. 

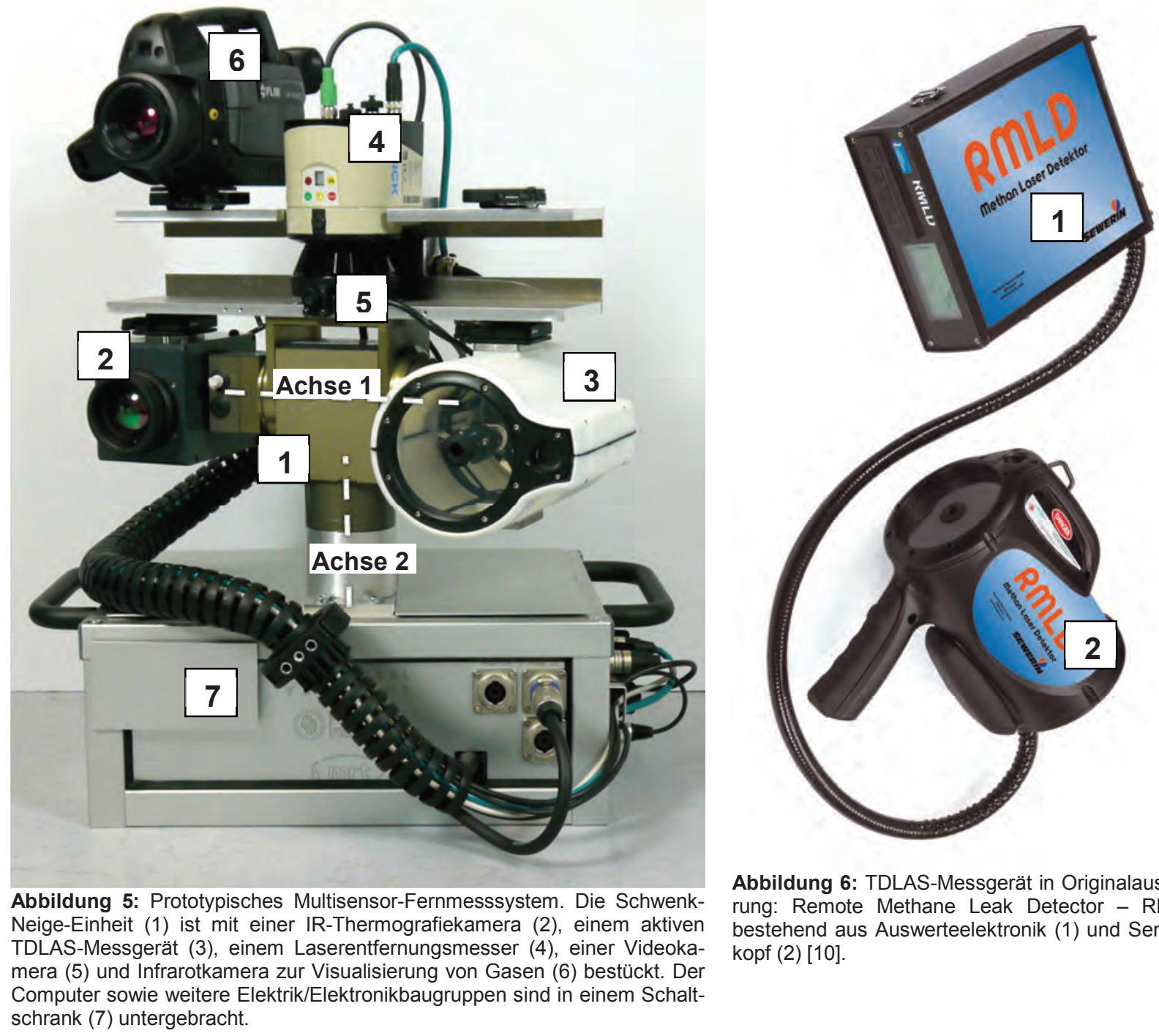

Abbildung 6: TDLAS-Messgerät in Originalausführung: Remote Methane Leak Detector - RMLD bestehend aus Auswerteelektronik (1) und Sensor$\operatorname{kopf}(2)[10]$. schrank (7) untergebracht.

Das RMLD ist ein tragbares, handgeführtes Gerät, welches aus einem Messkopf und einer in einem externen Gehäuse untergebrachten Auswerteelektronik besteht, die über ein steifes Kabel (zum Schutz der innenliegenden Glasfaserleitung) fest verbunden sind (Abbildung 6). Um den Messkopf computergesteuert schwenken und neigen zu können wurde ein neues Kunststoffgehäuse konstruiert und mittels Rapid Prototyping auf einem 3D Drucker gefertigt. Da der Kasten mit der Auswerteelektronik zu groß und zu schwer ist, um inn auf der PTU zu montieren, wurde dieser im Schaltschrank untergebracht. Durch einfache Tests wurde unter den Randbedingungen des steifen Kabels und der gewünschten Schwenkbereiche eine sinnvolle Platzierung ermittelt und mittels CAD optimiert. Zur Stabilisierung und zum Zusammenfassen der Energie- und Datenleitungen zwischen Messgeräten und Schaltschrank wurde ein bei Industrierobotern verbreitetes flexibles Kabelführungssystem verbaut.

Der Schaltschrank wurde in Profilbauweise selbst gefertigt, da am Markt kein passendes fertiges Produkt verfügbar war. Die Seitenplatten wurden mit Gummidichtlippen eingepasst. Um ausreichend Anschlussund Erweiterungsmöglichkeiten zu bieten wurden diverse wasserdichte Steckverbindungen vorgesehen: $3 \times$ USB, 4 x GigE Netzwerk, 4 x Strom (jeweils 5V, 12V und 24V), $2 \times$ RS232, 1x CAN. Als Steckverbindungen wurden aufgrund der geforderten Robustheit sowie hohen Staub- und Wasserschutzgrade (IP65) Komponenten aus dem Bereich der Bühnentechnik eingesetzt. Intern wurden die Anschlüsse über einen Gigabit Netzwerkswitch und zwei USB 2.0 Hubs mit einem kompakten Industrie-PC (Intel Core 172720QM, 8GB RAM) verbunden. Dieser PC übernimmt sämtliche Aufgaben wie die Steuerung der PTU sowie die Aufzeichnung und Auswertung der Messdaten. Mittels Gigabit Ethernet Netzwerkverbindung erfolgt die Kommunikation mit der Roboterplattform. Dadurch wird der Integrationsaufwand bei den Roboterplattformen auf ein Minimum reduziert, da neben der mechanischen Befestigungsmöglichkeit nur eine Spannungsversorgung und eine Netzwerkverbindung vorgesehen werden muss. Abbildung 7 zeigt den Einsatz auf drei verschiedenen im Projekt RoboGas ${ }^{\text {Inspector }}$ verwendeten Roboterplattformen. Der Einsatz ohne eine Roboterplattform ist ebenfalls möglich. 
Der mit dem Gesamtaufbau erreichbare Schwenk- und Neigebereich ist in Abbildung 8 zu sehen und liegt beim Schwenken bei $\pm 90^{\circ}$ und beim Neigen bei $\pm 50^{\circ}$ gegenüber dem Horizont. Damit lassen sich vom Boden aus z. B. Rohrbrücken oder auch Rohrgräben inspizieren. Im aktuellen Ausbauzustand hat das Modul einen typischen Gesamtenergiebedarf von ca. 130W und wiegt insgesamt ca. $30 \mathrm{~kg}$.
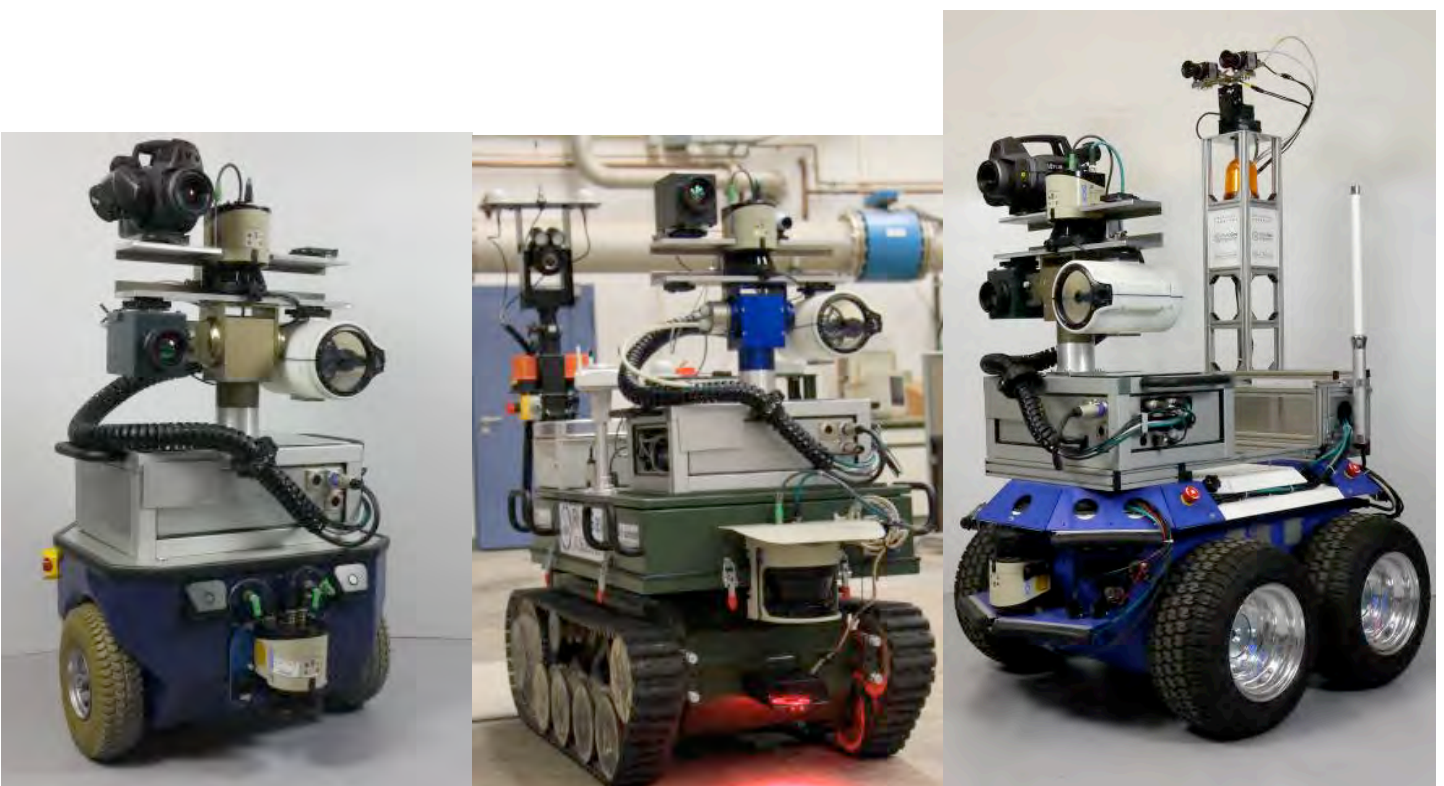

Abbildung 7: Inspektionsmodul auf verschiedenen Roboterplattformen. Links: Neobotix MP-S500, mitte: telerobtEODor, rechts: MobileRobotsSeekur Jr.

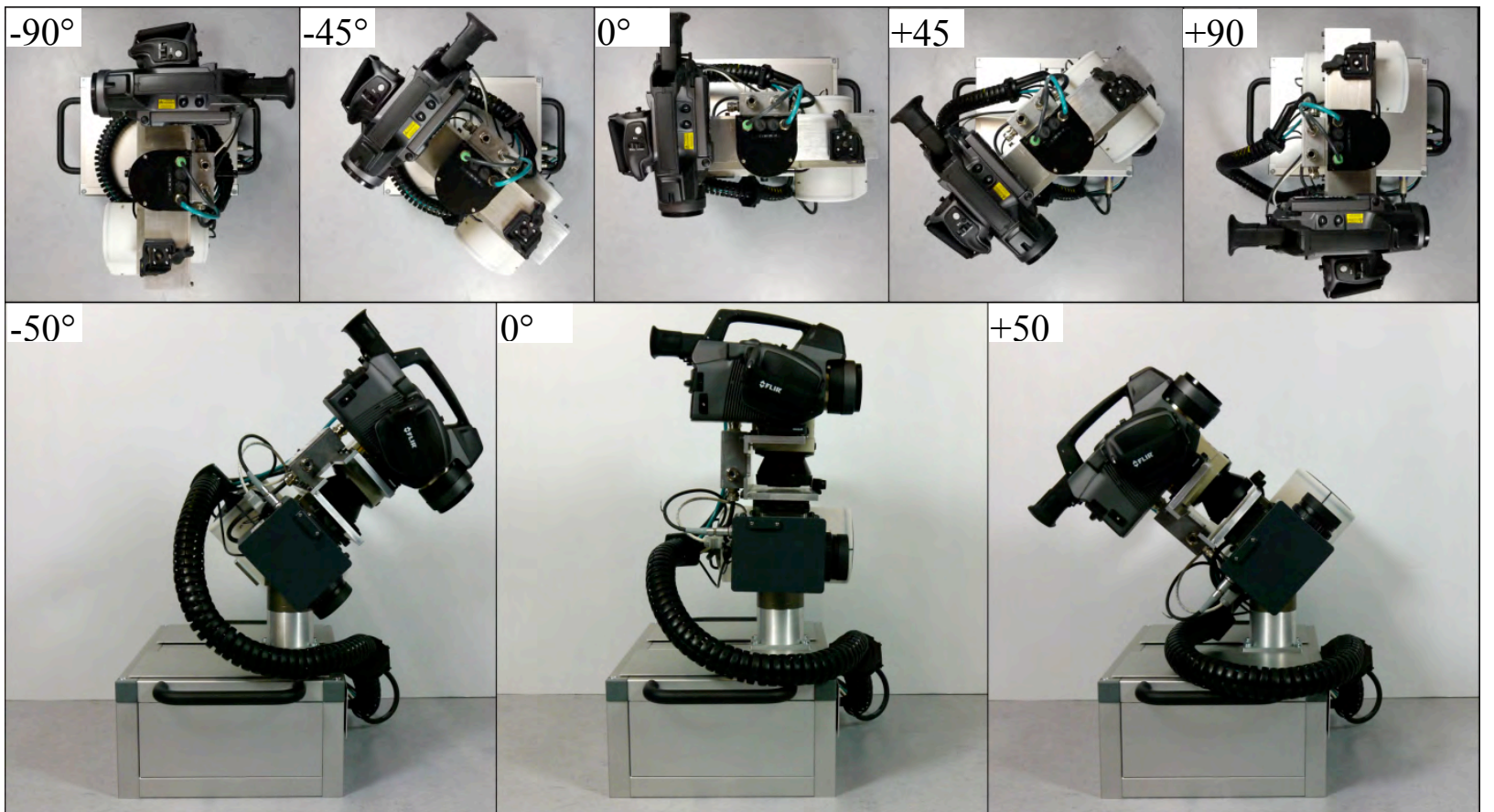

Abbildung 8:Schwenkbereich Draufsicht (oben) und NeigebereichSeitenansicht (unten) des Sensorsystems.

\section{Zusammenfassung und Ausblick}

In diesem Beitrag wurde ein Multisensor-Fernmesssystem zur Fluidleckerkennung beschrieben. Mit diesem System können Anlagenbereiche z. B. in petrochemischen Anlagen inspiziert werden, ohne dass die Messtechnik in den Gefahrenbereich eingebracht werden muss. Die verwendeten Messprinzipien nutzen Absorptionseffekte von elektromagnetischen Wellen zur direkten Detektion von Gasen sowie Wärmebildtechniken zur Erkennung von Sekundäreffekten (Expansionskälte, Verdunstungskälte).

Das System wurde für den Einsatz auf verschiedenen Roboterplattformen entworfen und erfolgreich getestet. Der kompakte Aufbau und die geringen elektrischen Anforderungen an die Trägerplattform (lediglich Stromversorgung und Netzwerkverbindung) sowie die bereits eingebaute Datenerfassungs- und 
Auswerteeinheit erlauben eine einfache Integration auf unterschiedlichen Robotern. Die großzügigen Schwenkbereiche sowie die schnellen Mess- und Rotationsgeschwindigkeiten erlauben ein gründliches und zügiges Inspizieren auch von größeren Bereichen.

Nach ersten erfolgreichen Tests sind Versuche im industriellen Umfeld (Raffinerie, Gasverdichterstation) geplant, bei denen sich sowohl die Messtechnik, als auch die Messstrategien behaupten müssen. KleinereÄnderungen und Anpassungen am Aufbau wiez. B. Spritzwasserschutz, Verbesserung der Kabelführung und Integration einer Innentemperaturüberwachung im Schaltschrank sind angedacht.

Das Projekt RoboGasInspector wird gefördert vom Bundesministerium für Wirtschaft und Technologie aufgrund eines Beschlusses des Deutschen Bundestages.

\section{Literatur}

[1] Universität Kassel, "RoboGasInspector - Projektziel," 2012, accessed 30. Jan.2012. [Online]. Available: www.robogasinspector.de

[2] IAEA (ed.) "Applicability of the leak before break concept."IAEA, Vienna, Rep. No. IAEA-TECDOC-710, 1993.

[3] H. Kaplan, Practical Applications of Infrared Thermal Sensing and Imaging Equipment. 3rd edition, SPIE, Bellingham, Washington, 2007.

[4] X. P. V. Maldague, Theory and practice of infrared technology for nondestructive testing. John Wiley \& Sons, Inc., New York, USA, 2001.

[5] G. Bonow, and A. Kroll, „Zur automatisierten Inspektion von Anlagen mittels Gasfernmesstechnik: Technologien und Geräte." In AUTOMATION 2011, Baden-Baden, 28.-29. Juni 2011.

[6] A. Kroll, "A survey on mobile robots for industrial inspections," in Proc. Intelligent Autonomous Systems 10 (IAS 10), Baden Baden, 23 - 25 Juli 2008, 406-414.

[7] Bundesministerium für Umwelt, Naturschutz und Reaktorsicherheit, „Technische Anleitung zur Verwertung, Behandlung und sonstigen Entsorgung von Siedlungsabfällen." Allgemeine Verwaltungsvorschrift Bundesrepublik Deutschland, 1993.

[8] S. Richter, "Verminderung diffuser Emissionen in der chemischen und Mineralölindustrie,"Texte 10/03, Berlin: Umweltbundesamt,2002.

[9] J. M. Hollas, Modern Spectroscopy. $4^{\text {th }}$ ed., Chichester: Wiley, 2003.

[10] Hermann Sewerin GmbH, "Sewerin RMLD," 2012,accessed 24. Jan.2012. [Online]. Available: www.sewerin.com/cms/produkte/gas/gaslecksuche-aussen/sewerin-rmld.html

[11] M. B. Frish,M. A. White, and M. G. Allen, "Handheld laser-based sensor for remote detection of toxic and hazardous gases," in Proc. Water, Ground and Air Pollution Monitoring (SPIE), Boston, MA, Nov. 2000, pp. 4199-4205.

[12] J. F. Tegstam, and R. Danjoux, "Gas leak detection in the oil and gas industry using infrared optical imaging," in Thermografie-Kolloquium 2007, Stuttgart, Sep. 2007, pp. 1-10.

[13] M. Vollmer, and K.-P.Möllmann, "IR imaging of gases: quantitative analysis,"in Proc. InfraMation, Vol 10, 2009.

[14] R. Benson, R. Madding, J. Lyons, and P. Czerepuszko, "Standoff passive optical leak detection of volatile organic compounds using a cooled InSb based infrared imager." in Proc. AWMA 99th Annual Meeting, New Orleans, LA, June, Paper 06-A-131, 2006.

[15] D. Furry, A. Richards, R. Lucier, R. Madding "Detection of Volatile Organic Compound (VOC) with a Spectrally Filtered Cooled Mid-Wave Infrared Camera." in Proc. InfraMation, Vol 6, 2005.

[16] FLIR Systems, Inc, "FLIR GF-320 Thermal Imaging Cameras", 2012, [Online; accessed 24-Jan.-2012]. Available: www.flir.com/cs/emea/en/view/?id=41384

[17] InfraTec GmbH, "WärmebildkameraVarioCAMhr head," 2012,accessed 24. Jan. 2012. [Online]. Available: www.infratec.de/de/thermografie/waermebildkameras/variocamr-hr-head-700-serie.html

[18] S. Soldan,G. Bonow, and A. Kroll,"RoboGasInspector - A Mobile Robotic System for Remote Leak Sensing and Localization in Large Industrial Environments: Overview and First Results."IFAC Workshop - Automatic Control in Offshore Oil and Gas Production, Norway, 2012, (submitted).

[19] W. Baetz, A. Kroll, and G. Bonow, "Mobile robots with active IR-optical sensing for remote gas detection and source localization," in Proc. IEEE Int. Conf. on Robotics and Automation (ICRA 2009), Kobe, Japan, 2009,pp. 2773-2778.

[20] W. Baetz, A. Kroll, and S. Soldan, "On Gas Leak Detection of Pressurised Components by Using Thermograms and Pattern Recognition Algorithms," in Proc. of the 8th International Conference on NDE in Relation to Structural Integrity for Nuclear and Pressurised Components, Berlin, Germany, 2010, pp. 503-512.

[21] A. Kroll, W. Baetz, and D.Peretzki, "On Autonomous Detection of Pressured Air and Gas Leaks Using Passive IRThermography for Mobile Robot Application."in Proc. IEEE Int. Conf. on Robotics and Automation (ICRA 2009), Kobe, Japan, 2009,pp. 921-926.

[22] SCHUNK GmbH \& Co. KG, "SCHUNK Rotary Modules PW," 2012, accessed 24. Jan. 2012, [Online]. Available: www.schunk-modular-robotics.com/left-navigation/service-robotics/components/actuators/rotary-modules/pw.html 